\title{
Effectiveness of Closed-PICU Perioperative Management of Children with Congenital Tracheal Stenosis
}

\author{
Kazunori Aoki ${ }^{1}$, Hiroshi Kurosawa ${ }^{1}$, Yusuke Seino ${ }^{1}$, Keiichi Morita ${ }^{1}$, Hironori Matsuhisa ${ }^{1}$, \\ and Yoshihiro Oshima ${ }^{1}$ \\ ${ }^{1}$ Hyogo Prefectural Kobe Children's Hospital
}

August 4, 2021

\begin{abstract}
Perioperative management of congenital tracheal stenosis (CTS) is challenging. Objectives: We investigated the effect of closed-pediatric intensive care unit (PICU) perioperative management by pediatric intensivists, compared with open-PICU management by surgeons, on the ventilator-free days (VFD) and length of postoperative PICU stay in children with CTS. Methods: This retrospective cohort study was conducted in a PICU in Japan. Children with CTS who underwent slide tracheoplasty were grouped according to whether they were perioperatively managed in an open (January 2015 to April 2016) or a closed (May 2016 to July 2019) PICU. Data were extracted from patient medical records. Results: In total, 13 and 38 patients were included in the open- and closed-PICU groups, respectively. Compared to the open-PICU group, the closed-PICU group had shorter durations of muscle relaxant administration (median: 4 vs. 5 days; $\mathrm{P}<0.001$ ); earlier initiation of enteral feeding (median: postoperative day 1 vs. postoperative day 7; P $<0.001$ ); more 28-day VFD (median: 21 vs. 20 days; P $=0.04$ ); and shorter duration of postoperative PICU stay (median: 16 vs. 36 days; $\mathrm{P}=0.002$ ), but the mortality did not differ significantly $(0 / 38[0 \%]$ vs. $1 / 13[8 \%] ; \mathrm{P}=0.25)$. Conclusions: Closed-PICU perioperative management with pediatric intensivists' participation significantly increased 28-day VFD and reduced the length of postoperative PICU stay in patients with CTS.
\end{abstract}

Original Research Article

Effectiveness of Closed-PICU Perioperative Management of Children with Congenital Tracheal Stenosis

Running title : Closed-PICU management of CTS

Kazunori Aoki, $\mathrm{MD}^{1, *}$; Hiroshi Kurosawa, $\mathrm{MD}^{1}$; Yusuke Seino, $\mathrm{MD}^{1}$; Keiichi Morita, $\mathrm{MD}^{2}$; Hironori Matsuhisa, $\mathrm{MD}, \mathrm{PhD}^{3}$; Yoshihiro Oshima, $\mathrm{MD}, \mathrm{PhD}^{3}$

1 Division of Pediatric Critical Care Medicine, Hyogo Prefectural Kobe Children's Hospital, 1-6-7, Minatojima-Minamimachi, Chuo-ku, Kobe, Hyogo, 650-0047, Japan

${ }^{2}$ Division of Pediatric Surgery, Hyogo Prefectural Kobe Children's Hospital 1-6-7, Minatojima-Minamimachi, Chuo-ku, Kobe, Hyogo, 650-0047, Japan

3 Division of Cardiovascular Surgery, Hyogo Prefectural Kobe Children's Hospital 1-6-7, MinatojimaMinamimachi, Chuo-ku, Kobe, Hyogo, 650-0047, Japan

* Corresponding author: Kazunori Aoki

Division of Pediatric Critical Care Medicine, Hyogo Prefectural Kobe Children's Hospital

1-6-7, Minatojima-Minamimachi, Chuo-ku, Kobe, Hyogo, 650-0047, Japan 
Phone: +81-78-945-7300

Fax: +81-78-302-1023

E-mail: aoki_kch@hp.pref.hyogo.jp

ORCID: https://orcid.org/0000-0002-7576-7607

\section{Abstract}

Perioperative management of congenital tracheal stenosis (CTS) is challenging.

Objectives: We investigated the effect of closed-pediatric intensive care unit (PICU) perioperative management by pediatric intensivists, compared with open-PICU management by surgeons, on the ventilator-free days (VFD) and length of postoperative PICU stay in children with CTS.

Methods: This retrospective cohort study was conducted in a PICU in Japan. Children with CTS who underwent slide tracheoplasty were grouped according to whether they were perioperatively managed in an open (January 2015 to April 2016) or a closed (May 2016 to July 2019) PICU. Data were extracted from patient medical records.

Results: In total, 13 and 38 patients were included in the open- and closed-PICU groups, respectively. Compared to the open-PICU group, the closed-PICU group had shorter durations of muscle relaxant administration (median: 4 vs. 5 days; $P<0.001$ ); earlier initiation of enteral feeding (median: postoperative day 1 vs. postoperative day 7; $P<0.001$ ); more 28 -day VFD (median: 21 vs. 20 days; $P=0.04$ ); and shorter duration of postoperative PICU stay (median: 16 vs. 36 days; $P=0.002$ ), but the mortality did not differ significantly $(0 / 38[0 \%]$ vs. $1 / 13[8 \%] ; P=0.25)$.

Conclusions: Closed-PICU perioperative management with pediatric intensivists' participation significantly increased 28-day VFD and reduced the length of postoperative PICU stay in patients with CTS.

Keywords: pediatric intensive care units; tracheal stenosis; perioperative care; surgical intensive care

\section{Introduction}

Congenital tracheal stenosis (CTS) is a rare disease that affects 1 in 64,500 births $^{1}$. It is characterized by the presence of complete tracheal rings, which can lead to narrowing of the airway ${ }^{1}$. Its clinical findings vary depending on the length and diameter of CTS. The disease may be fatal in some cases and is often exacerbated during infancy when developmental activity is increased, thereby requiring surgical treatment. CTS is associated with cardiovascular malformations in $80 \%$ of cases $^{1}$. Therefore, perioperative management requires close monitoring of respiration and circulation, and careful management in the pediatric intensive care unit (PICU) is required.

The surgical strategy for CTS varies depending on tracheal morphology, clinical symptoms, and the presence of cardiac complications ${ }^{2}$. Decision-making regarding the performance of simultaneous and staged tracheal and cardiac surgery is crucial $^{3}$ (Figure 1). Therefore, a collaboration of multiple departments, including cardiovascular and pediatric surgery, is warranted. Slide tracheoplasty is performed with the assistance of cardiopulmonary bypass (CPB), followed by PICU-based management for several days or weeks. Continuous muscle relaxants are prescribed postoperatively for several days to prevent excessive stress on the anastomosis.

In adults, A high-intensity intensive care unit (ICU), managed by intensivists on a daily basis, has been recommended (Grade 1B) ${ }^{4}$ and has been shown to increase patient survival, reduce length of ICU stay ${ }^{5}$, and decrease mortality in special disease groups such as those admitted to adult cardiac ICUs ${ }^{6}$. Moreover, PICU management by pediatric intensivists has been shown to reduce mortality, duration of mechanical ventilation, length of PICU stay, and hospital-acquired infections ${ }^{7}$.

Perioperative management of CTS at the Hyogo Prefectural Kobe Children's Hospital (hereafter referred to as "our hospital"), before May 2016, was performed by pediatric and cardiovascular surgeons because there 
were no pediatric intensivists employed by our hospital. Since the establishment of the new PICU in May 2016, the open-PICU management system in our hospital became a closed one, with pediatric intensivists present on-site 24 hours a day. This changed the perioperative management of patients with CTS.

In this study, we investigated the hypothesis that closed-PICU management by pediatric intensivists improved the outcomes of patients with CTS.

\section{Methods}

\subsection{Study design}

This single-center retrospective cohort study was conducted in a medical-surgical, 25-bed PICU in Japan between January 2015 and January 2019.

\subsection{Participants}

Patients with CTS who underwent slide tracheoplasty at our hospital between January 2015 and August 2019 were included in the study. Same surgeons worked throughout the study. Clinical data were collected before and after the establishment of the closed-PICU management system following the relocation of the hospital in May 2016 were retrospectively reviewed. Patients were categorized into two groups: an open-PICU group and a closed-PICU group. The open-PICU group underwent surgery between January 2015 and April 2016 and perioperative management was provided in an open PICU by cardiovascular and pediatric surgeons. The closed-PICU group underwent surgery between May 2016 and July 2019 and perioperative management was provided in a closed PICU by pediatric intensivists.

\subsection{Ethics}

This study was approved by the Institutional Review Board of Hyogo Prefectural Kobe Children's Hospital (No. R2-8; September 2, 2020). The requirement for informed consent was waived because of the retrospective design.

\subsection{Outcomes}

The following patient characteristics were examined: age, sex, height, weight, chromosomal abnormality, risk adjustment in congenital heart surgery ${ }^{8}$, pediatric Sequential Organ Failure Assessment score ${ }^{9}$ within 24 hours after surgery, age at diagnosis, duration from diagnosis to surgery, indication for surgery, mechanical ventilation management until the date of surgery, and disease background, which included presence of comorbid cardiac disease and total length of tracheal stenosis.

Surgery-related factors such as duration of the operation and CPB, presence of simultaneous cardiopulmonary and tracheal surgery, tracheal bifurcation plasty, administration of postoperative muscle relaxants, and timing of postoperative enteral feeding initiation were examined. The duration of $\mathrm{CPB}$ was influenced by the total intraoperative duration, including extracorporeal membrane oxygenation (ECMO), because surgery for tracheal stenosis alone can be performed under ECMO. Outcomes including anastomotic leakage, 28-day ventilator-free days (VFD), tracheostomy, cardiopulmonary resuscitation (CPR) events that occurred from the postoperative period to the first discharge, and mortality were evaluated. Anastomotic leakage was defined as tracheal leakage that required an additional surgical procedure at the anastomosis site.

\subsection{Management of congenital tracheal stenosis}

The open-PICU group was managed perioperatively by pediatric and cardiovascular surgeons, whereas the closed-PICU group was managed by pediatric intensivists. In addition to this major change in management, the following measures were taken by the pediatric intensivists in the closed-PICU group: (1) interdisciplinary conferences were held every morning to discuss any multispecialty issues; (2) daily levels of sedation and other aspects of patient care were shared at the patients' bedside with the nurse and intensivists in charge; (3) Intraoperative respiratory and circulation management problems during surgery and anesthesia were shared with intensive care using a standardized format; (4) resuscitation and ECMO trouble-shooting simulations were conducted regularly with nurses and medical technicians, and the team was educated not 
only in maintaining and managing emergency equipment but also in responding to emergency situations; (5) early rehabilitation involving respiratory physical therapy such as prevention of atelectasis and body positioning by physical therapists was initiated in the PICU on the day after the surgery; and (6) pediatric intensivists installed devices such as high-flow nasal cannula and non-invasive positive pressure, which were used expeditiously after extubation. In the open-PICU group, regular conferences were not held, anesthesiologists were not involved in sedation, and no sedation scale was used. ECMO simulation was mainly related to the initiation and instrumentation of ECMO by cardiovascular surgeons and nurses. It was not based on the resuscitation algorithms.

\subsection{Statistical analysis}

Fisher's exact test and the Mann-Whitney U test were used to analyze categorical and continuous variables, respectively. Analyses were performed using Stata/SE version 13.1 (Stata Corp., College Station, Texas). P values $<0.05$ were considered statistically significant.

\section{Results}

There were 51 eligible patients, of whom 13 and 38 were included in the open- and closed-PICU groups, respectively (Table 1). All patients underwent slide tracheoplasty. The median age and weight of the patients were 8 months (range: 1-118 months) and $6.5 \mathrm{~kg}$ (range: $2.3-20.8 \mathrm{~kg}$ ), respectively. Thirty-five of the 39 patients with comorbid cardiac conditions underwent simultaneous cardiovascular surgery. Pulmonary artery slings were the most common comorbid condition $(\mathrm{n}=24,47 \%)$. None of the patients underwent preoperative tracheostomy. Eleven patients (21\%) remained on preoperative mechanical ventilatory management because of difficulty in extubation due to tracheal stenosis.

More patients in the open-PICU group were intubated until surgery (6 [46\%] vs. $5[13 \%] ; P=0.02)$. There were no significant differences in other patient background characteristics or surgery-related factors. The duration of administration of muscle relaxants was shorter in the closed-PICU group (4 [3-10] vs. 5 [4-19] days; $P<0.001)$. Moreover, enteral feeding was started earlier in the closed-PICU group (1 [0-3] vs. 7 [1-24] postoperative days; $P<0.001$, Table 1$)$.

The outcomes evaluated are shown in Table 2. Anastomotic leakage $(1[8 \%]$ vs. $0[0 \%] ; P=0.25)$ and tracheostomy procedures $(2[15 \%]$ vs. $4[11 \%] ; P=0.62)$ were not significantly different between the two groups. However, the length of postoperative PICU stay was significantly shorter (16 [9-77] vs. 36 [16-183] days; $P=0.002)$ and the median number of 28 -day VFD (21 [0-23] vs. $20[0-22]$ days; $P=0.04)$ was significantly greater in the closed-PICU group. The number of CPR events $(2[15 \%]$ vs. $3[8 \%] ; P=0.59$ and mortality (1 $[8 \%]$ vs. $0[0 \%] ; P=0.25$ ) did not differ significantly between the two groups.

\section{Discussion}

Our findings suggest that closed-PICU perioperative management by pediatric intensivists results in a significant increase in the 28-day VFD and reduction in the length of postoperative PICU stay of CTS patients.

The median 28-day VFD was longer in the closed-PICU group than in the open-PICU group. This is likely to be attributable to the changes in the preoperative and postoperative respiratory management policies. The introduction of the sedation scale may have contributed to this result. In terms of preoperative respiratory management, more patients in the open-PICU group were intubated until surgery. Fewer patients underwent preoperative mechanical ventilatory management in the closed-PICU group due to inclusion of cases transferred from other hospitals, in whom extubation was difficult. However, they were successfully extubated in our hospital, thus allowing management in the PICU without a mechanical ventilator until surgery. In the closed-PICU group, we achieved a shortened duration of postoperative muscle relaxant administration by controlling the degree of sedation stringently using a newly introduced sedation scale. We also worked aggressively to ensure the use of appropriate ventilatory support devices to reduce the effort of breathing after extubation. These devices were used in $89 \%$ of patients in the closed-PICU group compared to only $39 \%$ of patients in the open-PICU group. In the perioperative management of CTS, we assumed that ventilator-induced diaphragmatic dysfunction ${ }^{10,11}$ was always present due to long-term 
preoperative mechanical ventilatory management, diaphragmatic immobilization secondary to postoperative use of muscle relaxants, and increased airway resistance due to postoperative tracheal edema. Furthermore, the increased respiratory rate and secretions due to crying increased the relative airway resistance, thereby resulting in an increase in respiratory effort. Sedation management was essential in preventing crying; however, the difficulties encountered in the removal of secretions due to over-sedation could have resulted in ventilation failure. These changes in perioperative respiratory management, including the introduction of a sedation scale and early rehabilitation, may have been the reasons behind the increase in the 28-VFD of the closed-PICU group.

The length of postoperative PICU stay was significantly shorter in the closed-PICU group. A prolonged PICU stay increases the risk for delirium in children ${ }^{12}$; hence, shortening ICU stay is beneficial to patients and may help curb their hospitalization costs. The presence of a pediatric intensivist in the PICU was associated with lower patient mortality, duration of mechanical ventilation, length of PICU stay, and hospitalacquired infections ${ }^{7}$. Additionally, a 24-hour shift of pediatric intensivists in the PICU are associated with reduction the duration of mechanical ventilation and length of ICU stay as compared with a 12hour shift ${ }^{13}$. Our study corroborated the finding that the 24 -hour shift of pediatric intensivists reduced the length of postoperative PICU stay. One possible explanation is the improved adherence to guidelines by the intensivists ${ }^{14}$. The guidelines of the European Society of Intensive Care Medicine ${ }^{15}$ recommend early initiation of enteral feeding in patients with controlled hypoxemia, hypercapnia, and acidosis. Furthermore, the guidelines also recommend against delays in the initiation of nutrition when using muscle relaxants. In this study, enteral feeding was started earlier in the closed-PICU group, suggesting that pediatric intensivists may have been managing patients in compliance with the guidelines.

Additionally, we trained the teams in resuscitation and ECMO trouble-shooting simulations to respond to emergency situations better. Perioperative management of CTS patients by a multidisciplinary team significantly reduces the length of PICU stay, thereby reducing the cost of treatment ${ }^{16}$. In this study, CPR events occurred in 10\% of the patients after CTS surgery. This suggested the importance of creating teams that were well trained in emergency response procedures such as extracorporeal cardiopulmonary resuscitation (ECPR). One case in each group of our study required postoperative ECPR; the patient in the open-PICU group died, whereas the one in the closed-PICU group survived. For conditions like CTS, where multiple professionals are involved and postoperative management or care is complex, management by a multidisciplinary team may have led to a shorter PICU stay. The mortality rate in our study (2\%) is comparable with those seen in previous reports $(5-13 \%)^{17-19}$. Furthermore, our results are comparable to those of a previous report by Butler et al. ${ }^{20}$, which has the largest study sample $(n=101)$. There were no deaths in the closed-PICU group, suggesting that our team was well trained and well equipped in responding to sudden events adverse such as postoperative ventilation failure and cardiac arrest.

CTS is a rare disease, and only a few large-scale studies have been published ${ }^{20,21}$. These studies have mainly evaluated surgical management, but, to the best of our knowledge, only one report ${ }^{16}$ focused on perioperative management. Only a few studies have demonstrated the benefits of being managed by a pediatric intensivist. These studies have studied heterogeneous populations because the target population has included all patients admitted to the PICU ${ }^{7,13}$. This study, to the best of our knowledge, is the first to observe the impact of pediatric intensivists on patient outcomes in a relatively large and homogeneous population despite the rare prevalence of CTS.

This study had some limitations. First, the sample size was small, and there were missing data in the open-PICU group. Nosocomial infections such as ventilator-associated pneumonia were important factors affecting patient outcomes; however, surveillance was not available in the open-PICU group. Additionally, data on detailed ventilator settings were not available in the medical records. The second limitation is the pre-post comparison design of this study. Changes in intraoperative management and improvement in surgical techniques have resulted in shortened duration of mechanical ventilation management and decreased mortality ${ }^{20,22}$. However, we attempted to minimize the impact of changes over time by limiting the study to patients undergoing slide tracheoplasty and by only including cases that had undergone surgery in the 
year prior to the change in PICU management in the open-PICU group. Finally, there was a lack of patient matching by severity of illness. Preoperative ECMO management ${ }^{20}$, low body weight, and younger age ${ }^{21}$ have been reported to be risk factors for death. Preoperative ECMO management was not performed in either group, and there were no significant differences in weight and age. The number of patients who had been intubated until surgery was significantly higher in the open-PICU group than in the closed-PICU group. This was probably related to the change in attempting extubation and enabling the growth of the patients until better operative conditions, as far as could be safely achieved, were available. In addition, details regarding bronchial morphology, such as the difference between the left and right bronchial diameters (bronchial mismatch) ${ }^{23}$ and surgical technique ${ }^{24}$, were not analyzed.

Additionally, the presence of confounding factors cannot be excluded due to the retrospective design of the study. Future prospective studies with more participants are recommended to overcome these limitations.

\section{Conclusions}

In our study, closed-PICU perioperative management by pediatric intensivists was significantly associated with increased 28-day VFD and reduced length of postoperative PICU stay in patients with CTS. Further research is needed to validate whether patient management by pediatric intensivists contributes to improved patient outcomes.

\section{Acknowledgments}

We would like to thank C. Miyakoshi, MD, MSc, DPH, for advice on the analysis method and reliability of the analysis results. We would like to thank Drs. N. Miyashita, Y. Nagai, T. Hasegawa, and T. Hatakeyama for their helpful discussions and comments on the manuscript.

Funding: None.

Conflict of Interest: None.

\section{Author Contributions}

Kazunori Aoki: conceptualization, data curation, formal analysis, investigation, writing - original draft

Hiroshi Kurosawa: conceptualization, methodology, writing - review \& editing

Yusuke Seino: conceptualization

Keiichi Morita: resources, data curation

Hironori Matsuhisa: writing - review \& editing

Yoshihiro Oshima: writing - review \& editing

Ethical approval: This study was approved by the Institutional Review Board of Hyogo Prefectural Kobe Children's Hospital (No. R2-8; September 2, 2020). The requirement for informed consent was waived because of the retrospective design.

Data Availability: The data that support the findings of this study are available from the corresponding author, KA, upon reasonable request.

\section{References}

1. Hewitt RJ, Butler CR, Maughan EF, Elliott MJ. Congenital tracheobronchial stenosis. Semin Pediatr Surg 2016;25:144-149.

2. Okamoto T, Nishijima E, Maruo A, Yokoi A, Takamizawa S, Satoh S, Oshima Y. Congenital tracheal stenosis: the prognostic significance of associated cardiovascular anomalies and the optimal timing of surgical treatment. J Pediatr Surg 2009;44:325-328. 
3. Maruo A, Oshima Y, Yoshida M, Shimazu C, Higuma T, Inoue T, Yamaguchi M. [Surgical repair of congenital heart disease associated with airway obstructive lesions.] Pediatric Cardiology and Cardiac Surgery 2010;26:375-383. [Article in Japanese]

4. Nates JL, Nunnally M, Kleinpell R, Blosser S, Goldner J, Birriel B, Fowler CS, Byrum D, Miles WS, Bailey H, et al. ICU admission, discharge, and triage guidelines: A framework to enhance clinical operations, development of institutional policies, and further research. Crit Care Med. 2016;44:1553-1602.

5. Ogura T, Nakamura Y, Takahashi K, Nishida K, Kobashi D, Matsui S. Treatment of patients with sepsis in a closed intensive care unit is associated with improved survival: a nationwide observational study in Japan. J Intensive Care 2018;6:57.

6. Na SJ, Chung CR, Jeon K, Park CM, Suh GY, Ahn JH, Carriere KC, Song YB, Choi JO, Hahn JY, et al. Association between presence of a cardiac intensivist and mortality in an adult cardiac care unit. J Am Coll Cardiol 2016;68:2637-2648.

7. Kesici S, Bayrakci B. Impact of leadership of trained intensivist in pediatric intensive care unit in a middle-income country: An uncontrolled before-after study. Indian J Pediatr 2020;87:262-267.

8. Jenkins KJ, Gauvreau K. Center-specific differences in mortality: preliminary analyses using the Risk Adjustment in Congenital Heart Surgery (RACHS-1) method. J Thorac Cardiovasc Surg 2002;124:97-104.

9. Matics TJ, Sanchez-Pinto LN. Adaptation and validation of a pediatric sequential organ failure assessment score and evaluation of the Sepsis-3 definitions in critically ill children. JAMA Pediatr 2017;171:e172352.

10. Supinski GS, Morris PE, Dhar S, Callahan LA. Diaphragm dysfunction in critical illness. Chest 2018;153:1040-1051.

11. Goligher EC, Brochard LJ, Reid WD, Fan E, Saarela O, Slutsky AS, Kavanagh BP, Rubenfeld GD, Ferguson ND. Diaphragmatic myotrauma: A mediator of prolonged ventilation and poor patient outcomes in acute respiratory failure. Lancet Respir Med 2019;7:90-98.

12. Traube C, Silver G, Reeder RW, Doyle H, Hegel E, Wolfe HA, Schneller C, Chung MG, Dervan LA, DiGennaro JL, et al. Delirium in critically ill children: An International Point Prevalence Study. Crit Care Med. 2017;45:584-590.

13. Nishisaki A, Pines JM, Lin R, Helfaer MA, Berg RA, Tenhave T, Nadkarni VM. The impact of 24-hr, in-hospital pediatric critical care attending physician presence on process of care and patient outcomes*. Crit Care Med 2012;40:2190-2195.

14. Gajic O, Afessa B, Hanson AC, Krpata T, Yilmaz M, Mohamed SF, Rabatin JT, Evenson LK, Aksamit TR, Peters SG, et al. Effect of 24-hour mandatory versus on-demand critical care specialist presence on quality of care and family and provider satisfaction in the intensive care unit of a teaching hospital. Crit Care Med 2008;36:36-44.

15. Blaser AR, Starkopf J, Alhazzani W, Berger MM, Casaer MP, Deane AM, Fruhwald S, Hiesmayr M, Ichai C, Jakob SM, et al. ESICM Working Group on Gastrointestinal Early enteral nutrition in critically ill patients: ESICM clinical practice guidelines. Intensive Care Med. 2017;43:380-398.

16. Kocyildirim E, Kanani M, Roebuck D, Wallis C, McLaren C, Noctor C, Pigott N, Mok Q, Hartley B, Dunne C, et al. Long-segment tracheal stenosis: slide tracheoplasty and a multidisciplinary approach improve outcomes and reduce costs. J Thorac Cardiovasc Surg 2004;128:876-882.

17. Hofferberth SC, Watters K, Rahbar R, Fynn-Thompson F. Management of congenital tracheal stenosis. Pediatrics 2015;136:e660-e669.

18. Speggiorin S, Torre M, Roebuck DJ, McLaren CA, Elliott MJ. A new morphologic classification of congenital tracheobronchial stenosis. Ann Thorac Surg 2012;93:958-961. 
19. Manning PB, Rutter MJ, Lisec A, Gupta R, Marino BS. One slide fits all: the versatility of slide tracheoplasty with cardiopulmonary bypass support for airway reconstruction in children. J Thorac Cardiovasc Surg 2011;141:155-161.

20. Butler CR, Speggiorin S, Rijnberg FM, Roebuck DJ, Muthialu N, Hewitt RJ, Elliott MJ. Outcomes of slide tracheoplasty in 101 children: a 17-year single-center experience. J Thorac Cardiovasc Surg 2014;147:1783-1789.

21. Yokoi A, Hasegawa T, Oshima Y, Higashide S, Nakatani E, Kaneda H, Kawamoto A, Nishijima E. Clinical outcomes after tracheoplasty in patients with congenital tracheal stenosis in 1997-2014. J Pediatr Surg 2018;53:2140-2144.

22. Matsushima S, Matsuhisa H, Morita K, Yokoi A, Maeda K, Oshima Y. Switch to extracorporeal membrane oxygenation during cardiac and tracheal repair. Ann Thorac Surg 2020;110:e181-e183.

23. Cetrano E, Trezzi M, Secinaro A, Di Chiara L, Trozzi M, Bottero S, Polito A, Carotti A. Bronchial mismatch as a predictor of respiratory failure after congenital tracheal stenosis repair. Ann Thorac Surg 2018;105:1264-1271.

24. Morita K, Yokoi A, Fukuzawa H, Hisamatsu C, Endo K, Okata Y, Tamaki A, Mishima Y, Oshima Y, Maeda K. Surgical intervention strategies for congenital tracheal stenosis associated with a tracheal bronchus based on the location of stenosis. Pediatr Surg Int 2016;32:915-919.

\section{Figure legend}

Figure 1. Treatment strategies for patients with congenital tracheal stenosis with cardiac complications in our hospital

CHD, congenital heart disease; OP, operation (surgery); TS, tracheal stenosis

${ }^{\text {a }}$ OP indication for TS: choking episode; history of tracheal intubation and mechanical ventilation; diameter of narrowest segment is less than $2 \mathrm{~mm}$

b Complicated repair: newborn or infant; complex cardiac repair; complex tracheal repair

${ }^{c}$ Stable ventilation: Tracheal intubation tube [?]3.0 mm

Reproduced from Maruo A, et al. ${ }^{3}$, with permission.

\section{Hosted file}

tables.docx available at https://authorea.com/users/429152/articles/532876-effectiveness-ofclosed-picu-perioperative-management-of-children-with-congenital-tracheal-stenosis 


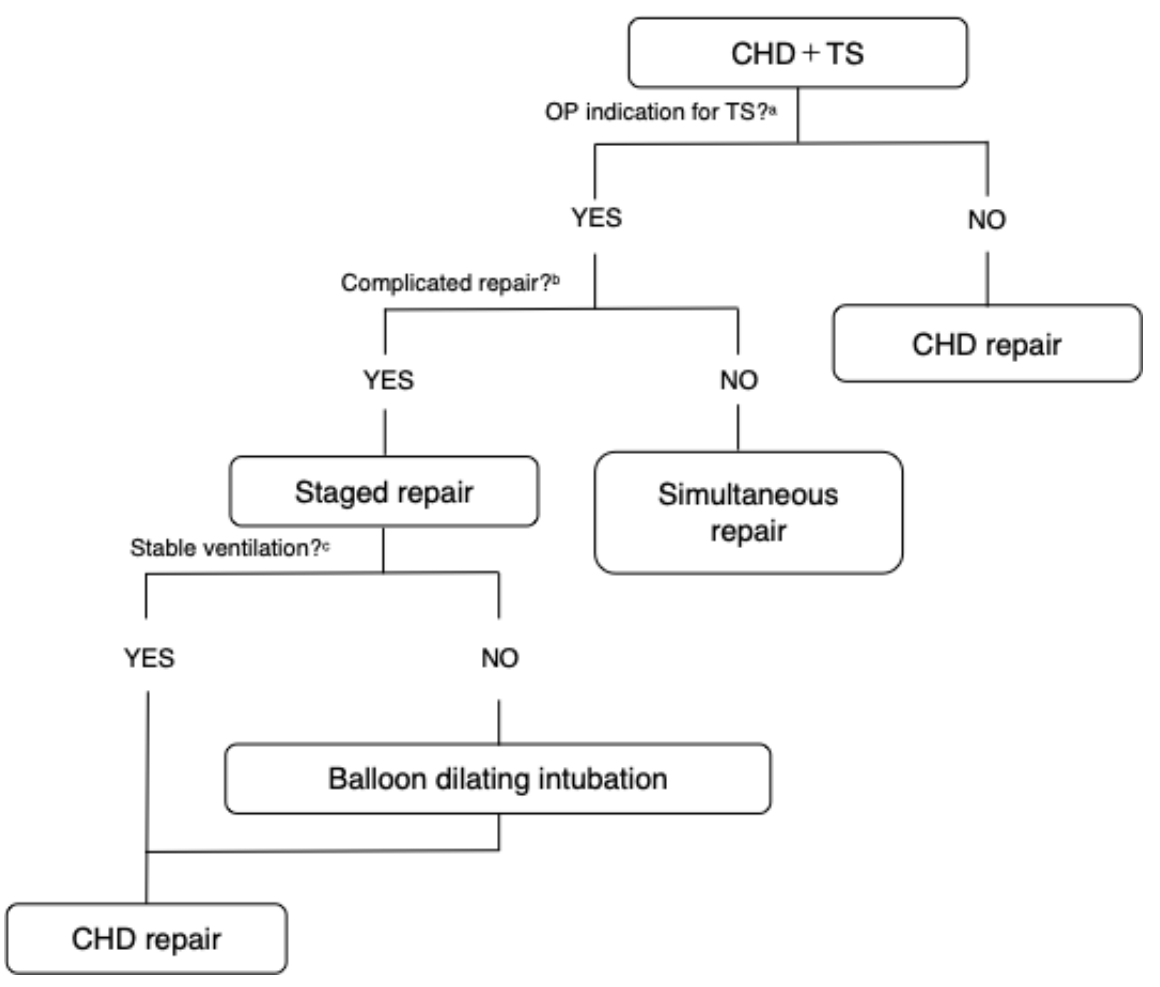

